\title{
A New Ranging Algorithm Based on NLOS Discrimination in Frequency Domain
}

\author{
Jing Li, Liang Pei, Maoyong Cao, and Nongliang Sun
}

\begin{abstract}
A new indoor ranging algorithm based on RootMin-Norm with the NLOS identification ability was proposed in this paper. We adopt Root-Min-Norm algorithm to estimate the time of arrival and received signal strength intensity of the first path firstly, then use the Friis model to decide if the first path is DLOS or not, and only if the FP is the DLOS path, we can use the TOA of the FP to estimate the T-R distance. A lot of experiment work was carried out to test the performance of the algorithm based on actual frequency channel response measured by vector network analyzer E5071B. Our experiment results show that the algorithm can realize accurate ranging with the mean ranging error $<0.6344 \mathrm{~m}$ and the standard bias $<0.7283 \mathrm{~m}$.
\end{abstract}

Index Terms-NLOS discrimination, ranging algorithm, TOA, Friis model.

\section{INTRODUCTION}

Indoor geolocation has attracted considerable attention in recent years, and many indoor geolocation systems has been developed [1]-[4]. Indoor geolocation mostly adopt RSSI and TOA methods. To the RSSI method, position fingerprint is often used, which depends on the deployment of the anchor nodes and indoor layout, and when the environment change, all the position fingerprint should be measured again, so finger print is not feasible in dynamic environments. In dynamic environments, TOA method is more feasible. However, the direct line of sight often cannot be detected because of the complex indoor environments, which called as NLOS conditions, and the NLOS is the most important factor degrades the accuracy of position in the TOA method.

In order to improve the performance of the TOA method in NLOS conditions, a lot of NLOS identification and mitigation algorithms were proposed. These algorithms can mainly be classified as two groups [5], [6]. One is to remove the NLOS error from the TOA measurement results, in which the NLOS error is estimated from large amount of history TOA measurement results. The other is to improve the robustness of the ranging algorithm to NLOS conditions, by using small weight values to the bases influenced by NLOS. ALL these NLOS mitigation methods are based on the statistics of the history measurement results, which is really difficult to achieve.

Manuscript received June 20, 2014; revised September 2, 2014.

Jing Li, Maoyong Cao, and Nongliang Sun are with the Qingdao Campus, Shandong University of Science and Technology, Qingdao, China (e-mail: lijing7261@sina.com, my_cao@263.net, nljackson@vip.163.com).

Liang Pei is with the Institute of Oceanographic Instrumentation, Shandong Academy of Sciences, Qingdao, China (e-mail: peiliang2002@163.com).
In this paper, we propose a new ranging algorithm with the NLOS identification ability based on super-resolution TOA estimation. We use the Root-Min-Norm algorithm to estimate the time of arrival of the first path (FP) that can be detected, and we have done a lot of research work in superresolution TOA estimation [7]-[9]. Then we compute the received signal strength (RSS) of the FP. And if the FP is the DLOS, TOA and RSSI must satisfy the Friis formula, then it can be conclude that only if the FP is the DLOS, the TOA of the FP and can be used for ranging purpose, otherwise, the FP we detected is NLOS, so can't be used for ranging.

\section{NLOS IDENTIFICATION BASED ON FRIIS MODEL}

Multipath effect is the most important feature of the indoor wireless channel, signal transmitted from the transmitter can arrive at the receiver at different times through reflection, refraction and diffraction besides the direct path, as shown in Fig. 1.

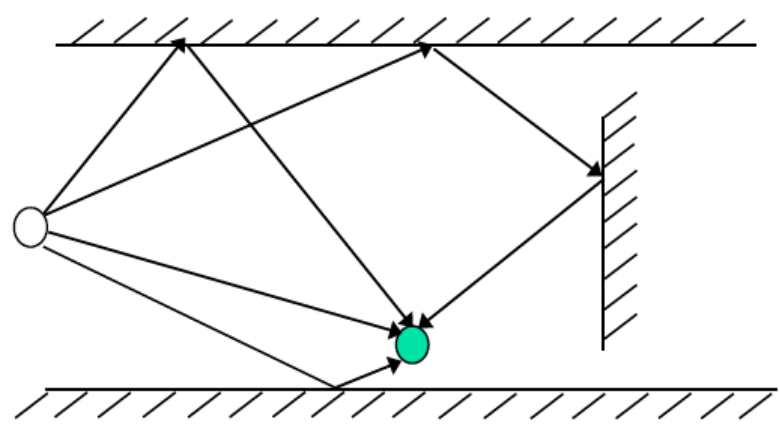

Fig. 1. Multipath effect.

So the channel impulse response can be modeled as the following:

$$
h(t)=\sum_{k=0}^{P-1} \alpha_{k} \delta\left(t-\tau_{k}\right)
$$

Here $P$ is the number of multi-path components, $\alpha_{k}=\left|\alpha_{k}\right| e^{j \phi_{n}},\left|\alpha_{k}\right|$ is the normalized signal strength, $\phi_{n}$ is the phase difference between the $k$ th and the DLOS path, and $\tau_{k}$ is the time of arrival of the $k$ th path.

If the FP is the DLOS path, that is, if we detected the DLOS path with the arrival time $\tau_{0}$ and received signal strength $\alpha_{0}$, then the distance between the transmitter and receiver is $C \tau_{0}$, here $C$ is the light speed. And the RSSI when $d=C \tau_{0}$ can be computed using the Friis formula. 
Friis formula is used to model the radio propagation in free space, in which there is no reflection, refraction and diffraction. So to the DLOS path, we can use the Friis formula to describe its received signal strength. Here we suppose that the transmitter transmits sphere wave, and the received signal strength with the distance $d$ from the transmitter can be described as the following:

$$
\begin{gathered}
P_{r}(d)=\frac{P_{t} G_{t} G_{r} \lambda^{2}}{(4 \pi)^{2} d^{2} L} \\
G=\frac{4 \pi A_{e}}{\lambda^{2}} \\
\lambda=\frac{c}{f_{c}}=\frac{2 \pi c}{w_{c}} \quad\left(c=3 \times 10^{8} \mathrm{~m} / \mathrm{s}\right)
\end{gathered}
$$

Here, $P t$ is the transmitted power, $P r$ is the received power, $G t$ is the antenna gain of the transmitter, $G r$ is the antenna gain of the receiver, $\mathrm{d}$ is the distance between the transmitter and receiver, $L$ is the loss coefficient independent of radio propagation $(L \geq 1), \lambda$ is the wavelength, and $c$ is the light speed.

So, we can estimate the TOA $\tau_{0}$ and RSS $\alpha_{0}$ of the FP firstly, then using formula (2) to compute the RSSI when the distance $\mathrm{d}=C \tau_{0}$ :

$$
P_{r}\left(d=c \tau_{0}\right)=\frac{P_{t} G_{t} G_{r} \lambda^{2}}{(4 \pi)^{2}\left(c \tau_{0}\right)^{2} L}
$$

Here, if $\alpha_{0}=P_{r}\left(c \tau_{0}\right)$, we can decide that the FP is the DLOS path. If $\alpha_{0}<P_{r}\left(c \tau_{0}\right)$, then the FP is not the DLOS path, and the FP must have been reflected, refracted or diffracted which will degrade the received energy in a large extent, and the direct path is obstructed, that is, it's the NLOS condition and we can't use the FP to estimate the distance between the transmitter and receiver.

\section{TOA AND RSS ESTIMATION BASED ON ROOT-MIN- NORM}

TOA is usually realized by time measurement directly in the time domain, in which we can't achieve high ranging accuracy which is limited to $1 / \mathrm{BW}$. Here, we will use the super-resolution TOA estimation to estimate that. And to the super-resolution algorithm, we have done a lot researching work. So we will describe the algorithm briefly here, if need more information, please check the reference [7]-[10].

By performing Fourier transform to the channel impulse response, we can get the frequency domain channel model:

$$
H(f)=\sum_{k=0}^{P-1} \alpha_{k} \exp \left(-j 2 \pi f \tau_{k}\right)
$$

Here, the most important is to estimate the arrival time of
$\mathrm{FP}$, that is $\tau_{0}$. And if FP is the DLOS, the distance can be estimated by $\tau_{0} \times c$, where $\mathrm{c}$ is the light speed. If we take $f$ in (2) as a time-domain variable and $\tau_{k}$ as a frequencydomain variable, then $H(f)$ can be seen as a time-domain signal consisting of $\mathrm{P}$ frequency components with the frequency $\tau_{k}$. That is, the problem of $\tau_{k}$ estimation now becomes a frequency estimation problem, so we can use the time-domain super-resolution spectral estimation theory to realize $\tau_{k}$ estimation. And there are several algorithms to realize super-resolution spectral estimation such as Prony, MUSIC, ESPIRIT and MIN-NORM. In this paper, we adopt the root-Min-norm method.

In order to achieve super-resolution TOA estimation, we sample the frequency channel response $H(f)$ at $N$ equally spaced frequencies in the frequency band $\left[f_{0}, f_{0}+B W\right]$, then we get the discrete frequency channel response samples: $y(i), i=0,1, \ldots, N-1$. We describe $y(i)$ as the following:

$$
y(i)=H(i)+e(i)=\sum_{k=0}^{P-1} \alpha_{k} \exp \left[-j 2 \pi\left(f_{0}+i \Delta f\right) \tau_{i}\right]+e(i)(7)
$$

Here $e(i)$ is the zero-mean AWGN noise with the variance $\sigma^{2}$. And the signal model in (7) can be described in vector form as formula (8):

$$
Y=H+E=S a+E
$$

where

$$
\begin{gathered}
Y=[y(0) y(1) \cdots y(N-1)] \\
H=[H(0) H(1) \cdots H(N-1)] \\
S=\left[s\left(\tau_{0}\right) s\left(\tau_{1}\right) \cdots s\left(\tau_{P-1}\right)\right] \\
s\left(\tau_{k}\right)=\left[1 \exp \left(-j 2 \pi \Delta f \tau_{k}\right) \cdots \exp \left[-j 2 \pi(N-1) \Delta f \tau_{k}\right]\right]^{T} \\
a=\left[\alpha_{0} \alpha_{1} \cdots \alpha_{L-1}\right]^{T} \\
E=[e(0) e(1) \cdots e(N-1)]
\end{gathered}
$$

Then we can compute the auto-correlation matrix of $Y$ :

$$
R_{Y Y}=E\left[Y Y^{H}\right]=S P S^{H}+\sigma^{2} I
$$

where $P=E\left\{a a^{H}\right\}$. The eigen-decomposition of $R_{Y Y}$ is as the following:

$$
R_{Y Y}=U \sum V^{H}
$$

Here $\sum=\operatorname{diag}\left[\lambda_{1} \lambda_{2} \cdots \lambda_{L}\right], \lambda_{1} \geq \lambda_{2} \geq \cdots \geq \lambda_{L} \geq$ $\lambda_{P+1}=\lambda_{P+2} \cdots=\lambda_{N}=\sigma^{2}$, is a diagonal matrix. We can 
divide the eigen values into signal exigent values and noise exigent values that is, the largest $P$ eigen-values is the related to the $P$ components of the signal, and the other $N-P$ eigenvalues is related to the AWGN noises. So the eigenvectors can also be divided into two groups, that is, signal eigenvectors and noise eigenvectors. Here, the subspace the signal eigenvectors span is called signal subspace ( $S_{\text {signal }}$ ), and the subspace the noise eigenvectors span is called noise subspace ( $S_{\text {noise }}$ ).

Then we can realize super-resolution TOA estimation based on the orthogonal signal subspace and noise subspace. And there are several algorithms we can use, here we adopt the Root-Min-Norm algorithm to solve the $\tau_{i}$. The MinNorm algorithm is based on the eigen vector $q$ with the minimum norm, and the detailed solving process can be found in reference [11]. Given the min-norm eigenvector $q$, to any vector $s(\tau)$ in $S_{\text {signal }}$, each $\tau_{i}$ must satisfy the following equation:

$$
s(\tau)^{H} q=0
$$

Then let $z=e^{j 2 \pi \Delta f \tau}$, we have:

$$
q_{i}(0)+q_{i}(1) z^{-1}+\cdots+q_{i}(m-1) z^{-(m-1)}=0
$$

It can be proved that equation (6) has exactly $\mathrm{P}$ roots [2], so after the $P$ roots is solved:

$$
z_{k}=e^{j 2 \pi \Delta f \tau_{k}}
$$

the $P$ frequencies can be estimated by:

$$
\hat{\tau}_{k}=\angle z_{k} / \Delta f 2 \pi
$$

And this is super-resolution TOA estimation based on Root-Min-Norm. And here the most important is the TOA of the FP:

$$
\hat{\tau}_{0}=\angle z_{0} / 2 \Delta f \pi
$$

What's more, the amplitude, that is, the received signal strength can also be estimated by non-linear least squre (NLS) as the following:

$$
\hat{\alpha}=\left|\left(A^{H} A\right)^{-1} A^{H} Y(n)\right|
$$

So the amplitude of the FP is: $\hat{a}_{0}$

Given $\hat{a}_{0}, \hat{\tau}_{0}$, we can use the Friis formula to identify the FP is DLOS or NLOS. Firstly, we know the propagation distance of the FP is:

$$
d_{0}=c \hat{\times} \tau_{0}
$$

Then using formula (5) to compute the RSS of FP, in formula (5), $\frac{P_{t} G_{t} G_{r} \lambda^{2}}{(4 \pi)^{2} L}$ is a constant, and we use $G$ to represent it, so Friis formula can be described as:

$$
P_{r}(d)=\frac{G}{d^{2}}
$$

And the value $G$ can be decided in actual measurements. So, if the following equation is satisfied:

$$
\hat{\alpha}_{0}=P_{r}\left(d_{0}\right)=\frac{G}{\left(c \cdot \tau_{0}^{2}\right)^{2}}
$$

We can conclude that the FP is DLOS. If not, the FP can't be the DLOS path, and can't be used in ranging. This is the new ranging algorithm based on NLOS identification in domain proposed in this paper.

\section{EXPERIMENTS}

The NLOS identification algorithm is realized in frequency domain, so we use vector network analyzer Agilent E5071B to obtain the frequency channel response. Agilent E5071B covers the $300 \mathrm{~K}-8.5 \mathrm{GHz}$ band. In our experiments, a sending antenna was connected to port1 of Agilent E5071B, and the receiving antenna was connected to port2 with a low loss wire.

By moving the receiving antenna to different locations, different frequency channel response can be measured between the two antennas after calibration. In order to test the performance of the NLOS identification algorithm, large amounts of measurements were carried out in different conditions (in different rooms or corridors with different Transmitter-Receiver distances and different obstacles).

Fig. 2 is the one of the channel responses measured by AgilentE5071B in the frequency band: [1.4GHz, $1.6 \mathrm{GHz}$, with the distance between the transmitting antenna and receiving antenna $5.1 \mathrm{~m}$.

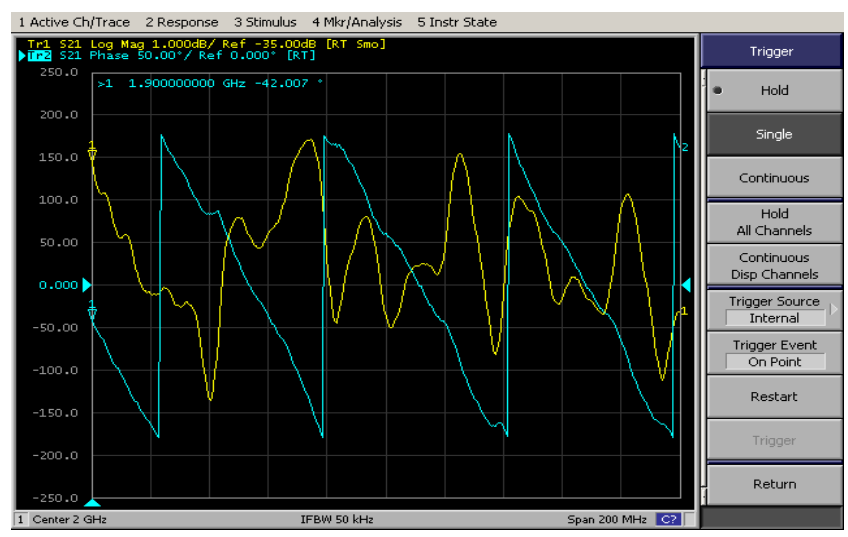

Fig. 2. Frequency channel response with the T-R distance $5.1 \mathrm{~m}$.

In Fig. 2, curve 1 is the amplitude response and curve 2 is the phase response. We can see the two curves is both obviously periodic, corresponding to formula (6), that is, the channel response we measured proves that the frequency 
channel response can be taken as a signal composed by several periodic components.

Based on the frequency channel response, we can use the Min-Norm algorithm to realize super-resolution TOA and RSS estimation. We apply different bandwidth to the RootMin-norm algorithm, that is, we use different bandwidth from $20 \mathrm{MHz}$ to $200 \mathrm{MHz}$ to estimate the distance between the transmitter and receiver. And the testing results are shown in Table I. From Table I we can see that precise ranging can be realized using the algorithm proposed in this paper.

In indoor geolocation systems, WLAN is often adopted as the physical network to realize indoor geolocation, and WLAN can offer $20 \mathrm{MHz}$ bandwith. So in our experiments, the length of the frequency window of each frequency channel response is also 20MHZ. We sample the frequency channel response in the $20 \mathrm{MHz}$ bandwidth with the frequency interval $0.5 \mathrm{MHz}$, then we can obtain 41 samples, that is, $N=41$. Then we can use the sample series to estimate the auto-correlation matrix, compute the signal eigenvectors and noise eigenvectors, then use Min-Norm algorithm to estimate TOA and RSS, finally use formula (18) to decide DLOS or NLOS. Our experiments includes three frequency bands (GHz): [1.49,1.51], [1.99,2.01], [2.99,3.01], and in each band we carried out 100 different measurements in different conditions, then we obtain the statistics of the TOA estimation as Table II From Table II we can conclude that accurate ranging can be realized by the Min-norm algorithm.

TABLE I: RANGING ERROR WITH DIFFERENT BANDWIDTH
\begin{tabular}{|c|c|c|}
\hline T-R $(\mathrm{m})$ & $\begin{array}{l}\text { Mean of ranging } \\
\text { error }(\mathrm{m})\end{array}$ & $\begin{array}{l}\text { Standard bias of } \\
\text { ranging error }(\mathrm{m})\end{array}$ \\
\hline 18 & 0.4407 & 0.2922 \\
\hline $18(1)$ & 0.5486 & 0.3006 \\
\hline $18(2)$ & 0.2777 & 0.3004 \\
\hline 15 & 0.0632 & 0.2992 \\
\hline $15(1)$ & 0.1047 & 0.4008 \\
\hline $15(2)$ & 0.0465 & 0.5098 \\
\hline 9 & 0.2279 & 0.4272 \\
\hline 6 & 0.1477 & 0.4450 \\
\hline 5 & 0.2901 & 0.3385 \\
\hline 4 & 0.2808 & 0.3104 \\
\hline 3 & 0.1609 & 0.4926 \\
\hline 2 & 0.3626 & 0.7199 \\
\hline 1 & 0.0074 & 0.2109 \\
\hline
\end{tabular}

TABLE II: RANGING ERROR WITH 20MHZ BANDWIDTH IN DIFFERENT

\begin{tabular}{|c|c|c|}
\hline Frequency band & $\begin{array}{c}\text { Mean of ranging } \\
\text { error(m) }\end{array}$ & $\begin{array}{c}\text { Standard Bias of } \\
\text { ranging error(m) }\end{array}$ \\
\hline$[1.49 \mathrm{GHz}, 1.51 \mathrm{GHz}]$ & 0.6344 & 0.2043 \\
\hline$[1.99 \mathrm{GHz}, 2.01 \mathrm{GHz}]$ & 0.4291 & 0.3878 \\
\hline$[2.99 \mathrm{GHz}, 3,01 \mathrm{GHz}]$ & 0.45 & 0.7283 \\
\hline
\end{tabular}

\section{CONCLUSION}

A new indoor ranging algorithm based on Root-Min-
Norm with the NLOS identification ability was proposed in this paper. We adopt Root-Min-Norm algorithm to estimate the time of arrival and received signal strength intensity of the first path firstly, then use the Friis model to decide if the first path is DLOS or not, that is, only if the FP is the DLOS path, we can use the time of arrival of FP to estimate the T$\mathrm{R}$ distance. We carried out a lot of testing work to test the performance of the algorithm, and the tests are all based on actual frequency channel response measured by vector network analyzer E5071B. Our experiment results show that accurate ranging can be realized with the mean ranging error $<0.6344 \mathrm{~m}$ and the standard bias $<0.7283 \mathrm{~m}$. This algorithm can also be used in many fields such as mobile robot navigation, ubiquitous computing, intelligent building, and so on.

\section{REFERENCES}

[1] Q. Yang, Y. Chen, J. Yin et al., "LEAPS: A location estimation and action prediction system in a wireless lan environment," in Proc. NPC, LNCS 3222, Wuhan, China, 2004, pp. 584-591.

[2] J. Schiller and A. Voisard, Location-Based Services, Morgan Kaufmann Publishers, 2004.

[3] S. H. Fang, T. N. Lin, and K. C. Lee, "A novel algorithm for multipath fingerprinting in indoor WLAN environments," IEEE Transactions on Communications, vol. 7, no. 9, pp. 3579-3588, 2008.

[4] P. J. Voltz and D. Hernandez, "Maximum likelihood time of arrival estimation for real-time physical location tracking of $802.11 \mathrm{a} / \mathrm{g}$ mobile stations in indoor environments," in Proc. Position Location and Navigation Symposium, 2004, pp. 585-591.

[5] M. P. Wylie and J. Holtzman, "The Non-Line of sight problem in mobile location estimation," IEEE ICUPC1996, vol. 2, pp. 827-831.

[6] Z. Xiao, B. X. Song, W. Sun, Q. Yu, and K. C. Yi, "NLOS identification based on statistic analysis of UWB localization error," Journal of Jilin University (Engineering and Technology Edition), vol. 40, no. 5, pp. 1381-1385, Sep. 2010.

[7] J. Li, "Research on the UHF radio wave propagation and location algorithms in mine tunnels (D)," Tianjin University, 2006.

[8] J. Li, L. Pei, M. Y. Cao, and N. L. Sun, "Super-resolution time of arrival estimation for indoor geolocation based on IEEE $802.11 \mathrm{a} / \mathrm{g}$," in Proc. WCICA08, 2008, pp. 6612-6615.

[9] J. Li, L. Pei, M. Y. Cao, and N. L. Sun, "Resolution analysis of the mmusic-based algorithm," in Proc. ICITIS, 2010, pp. 718-721.

[10] J. Li, "Frquency Domain TOA estimation Algorithm based on CrossHOC," Journal of Measurement Science and Instrumentation, vol. 2, no. 2, Feb. 2011.

[11] H. Saarnisaari. A postgraduate course: spectrum estimation (spectral analysis). (December 11-20, 2001). [Online]. Available: http://ee.oulu.fi/ harza/spect_estim/contents.html.

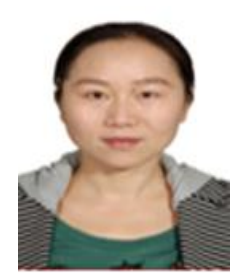

Jing Li was born in Dongyang, Zhejiang, on Oct. 27, 1978. She received the B.S. degree in electrical technology in 2000, and M.S degree in power electronics and power transmission from Shandong University of Science and Technology. Then she received the Ph.D. degree in optical engineering from Tianjin University.

She works in Shandong University of Science and Technology as a teacher now. Her research interests are wireless location, indoor geolocation, and signal processing.

Dr. Jing $\mathrm{Li}$ is one of the recipients of Scientific and Technological Award of Shandong province. 